\title{
RESTRICTION TO A PARABOLIC SUBGROUP AND IRREDUCIBILITY OF DEGENERATE PRINCIPAL SERIES OF $\operatorname{Sp}(2, \boldsymbol{C})$
}

\author{
BY KENNETH I. GROSS ${ }^{1}$
}

\author{
Communicated by Ray A. Kunze, June 12, 1970
}

A series of unitary representations of a semisimple Lie group is said to be a principal series if the representations are induced from characters of a so-called parabolic subgroup. In the event that the parabolic subgroup is minimal (i.e., a Borel subgroup) one says that the series is nondegenerate. It has recently been shown through the efforts of Kostant and Wallach, among others, that for the complex semisimple groups all of the representations in the nondegenerate principal series are irreducible. However, at present no such general result is known for the remaining series; i.e., the degenerate principal series.

This note considers the degenerate principal series of the rank two complex symplectic group $G=\operatorname{Sp}(2, C)$. $G$ has exactly two such series, each associated with a maximal parabolic subgroup which turns out to be a semidirect product of a normal nilpotent group with a subgroup isomorphic to the $2 \times 2$ complex general linear group $\operatorname{GL}(2, \boldsymbol{C})$. In one case the nilpotent group is abelian, and quite elementary classical Fourier analysis leads to a proof that the representations in the corresponding degenerate series are already irreducible when restricted to the parabolic subgroup. For the other case the analysis is much different, since the nilpotent group is nonabelian and the restriction is not irreducible.

In what follows we investigate this latter situation. In particular, we develop the required nonabelian harmonic analysis, use it to decompose the restrictions of the representations, and show that the representations of $G$ in this degenerate principal series are indeed not all irreducible. In this context one also obtains a natural variant of the Shale-Weil representation for the complex rank two special linear group $\mathrm{SL}(2, \mathrm{C})$.

In a paper in preparation we extend these results to the rank $n$ real and complex case and develop for these degenerate principal

AMS 1968 subject classifications. Primary 2257, 2265; Secondary 4790, 4428.

Key words and phrases. Symplectic group, principal series of representations, nilpotent group, harmonic analysis, Plancherel, commuting algebra, irreducible.

1 This research was supported in part by National Science Foundation Grant GP-11910. 
series the Kunze-Stein theory of intertwining operators, analytic continuation, uniformly bounded representations, and complementary series.

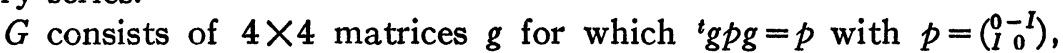
$I$ being the $2 \times 2$ identity matrix. The parabolic subgroup $H$ we consider is the semidirect product of the normal nilpotent subgroup $Z$ of matrices

$$
z=z(u, \xi)=(u, \xi)=\left(\begin{array}{cccc}
1 & 0 & 0 & 0 \\
-u_{2} & 1 & 0 & 0 \\
\xi & u_{1} & 1 & u_{2} \\
u_{1} & 0 & 0 & 1
\end{array}\right)
$$

where $u=\left(u_{1}, u_{2}\right) \in C^{2}$, with the subgroup $C$, isomorphic to $\operatorname{GL}(2, C)$, consisting of the matrices

$$
c=c(a, \alpha)=\left(\begin{array}{llll}
\alpha & 0 & 0 & 0 \\
0 & a_{11} & 0 & a_{12} \\
0 & 0 & \alpha^{-1} & 0 \\
0 & a_{21} & 0 & a_{22}
\end{array}\right)
$$

with $\alpha \in C^{*}$ (the multiplicative group of the complex field) and $a \in \operatorname{SL}(2, \boldsymbol{C})(=\operatorname{Sp}(1, \boldsymbol{C}))$.

We briefly describe the realization of the representations $T(\cdot, \chi)$ in the degenerate principal series which is appropriate to our analysis. Let $h=c z$ denote the generic element of $H$; let $W$ be the group transpose to $Z$ with generic element $w={ }^{t} z ; K=W C$ the group transpose to $H$ with generic element $k=w c$; and $X=K Z . X$ is an open set of full Haar measure, from which it follows that for each $g \in G, z g \in X$ for a.e. $z \in Z$; i.e.,

$$
z g=[w(z g) c(z g)] z \tilde{g}
$$

where $z \tilde{g} \in Z, w(z g) \in W$, and $c(z g)=c(a(z g), \alpha(z g)) \in C .(z, g) \rightarrow z \tilde{g}$ is a group action of $G$ on $Z$ in the sense that given $g_{1}, g_{2} \in G$ one has $\left(z \tilde{g}_{1}\right) \tilde{g}_{2}=z\left(g_{1} g_{2}\right) \sim$ for a.e. $z$. The representations $T(\cdot, \chi)$ are multiplier representations of $G$ in the space $\mathfrak{S}=L^{2}(Z)$, indexed by the unitary characters $\chi$ of $C$ where $\chi(c)=|\alpha|^{s}[\alpha]^{m}$ with $s$ purely imaginary, $m$ an integer, and $[\alpha]=\operatorname{sgn} \alpha=\alpha /|\alpha|$ for $\alpha \in C^{*}$. Then for $f \in \mathfrak{S}$ and $g \in G$,

$$
(T(g, \chi) f)(z)=|\alpha(z g)|^{s+4}[\alpha(z g)]^{m} f(z \tilde{g}) \quad \text { a.e. }
$$

The two special cases of this formula that we use below are as follows: 
For $h_{0}=c_{0} z_{0} \in H$,

$$
\left(T\left(h_{0}, \chi\right) f\right)(z)=\left|\alpha_{0}\right|^{s+4}\left[\alpha_{0}\right]^{m} f\left(\alpha_{0} u a_{0}+u_{0}, \alpha_{0}^{2} \xi+\xi_{0}+\alpha_{0}\left\langle u a_{0} \mid u_{0}\right\rangle\right)
$$

where $\langle u \mid v\rangle$ denotes the skew-form $u p^{t} v$ on $C^{2}$; and

$$
(T(p, \chi) f)(z)=|\xi|^{-(\bullet+4)}[-\xi]^{-m} f\left(z\left(-\frac{u_{2}}{\xi}, \frac{u_{1}}{\xi} ;-\frac{1}{\xi}\right)\right) .
$$

Theorem 1. Define the operator $J$ on Schwartz-class functions $f$ on $Z$ by

$$
(J f)(z(u, \xi))=-\int_{C^{2}}\left(\Delta_{3} f\right)(v, \xi+\langle u \mid v\rangle) d v
$$

where $\Delta_{3}$ denotes the Laplacian applied to the third coordinate; i.e., $\xi . J$ extends to a unitary operator on $\mathfrak{S}$ which together with the identity operator forms a basis for the commuting algebra of the restriction to $H$ of $T(\cdot, \chi)$ for all $\chi$.

COROLLARY 2. $T(\cdot, \chi)$ is irreducible for each $\chi \neq 1$ and is reducible for $\chi=1$.

The corollary follows from the theorem by means of two observations: (1) $p$ and $H$ generate $G$, and (2) $J$ fails to commute with $T(p, \chi)$ for $\chi \neq 1$ and commutes for $\chi=1$. As for the theorem, one can formally verify that $J$ commutes with $T(h, \chi)$ for all $h \in H$, but to see that $J$ is unitary and that $I$ and $J$ form a basis for the commuting algebra one introduces the Plancherel transform of the group $Z$. We outline the main ideas because they are of independent interest.

The following is a variant of the well-known Stone-von Neumann theorem:

THEOREM 3. (i) Exclusive of the one-dimensional representations, the dual object $\hat{Z}$ of $Z$ is $C^{*}$, and for each $\lambda \in C^{*}$ there corresponds a representation $U(\cdot, \lambda)$ of $Z$ in $\mathfrak{M}=L^{2}(\mathbf{C})$ defined for $f \in \mathfrak{M}$ by

$$
(U(z, \lambda) f)(t)=\exp \left\{i \operatorname{Re} \lambda\left(\xi-u_{2}\left(u_{1}+2 t\right)\right)\right\} f\left(t+u_{1}\right) .
$$

(ii) The map $\phi \rightarrow \hat{\phi}$ initially defined for $\phi$ in Schwartz' class on $Z$ by $\hat{\phi}(\lambda)=\int_{z} \phi(z) U(z, \lambda) d z$, extends to a unitary map $\mathfrak{F}$ of $\mathfrak{W}$ onto the Hilbert space $\hat{\mathfrak{S}}$ of functions on $\hat{Z}$ with values in Hilbert-Schmidt operators on $\mathfrak{M}$ which are square-summable with respect to Plancherel measure $d m(\lambda)=c|\lambda|^{2} d \lambda$ (where $c$ is a constant which we ignore). By means of the identification of $\hat{\phi}(\lambda)$ with its Hilbert-Schmidt kernel $\tilde{\phi}(\cdot, \cdot, \lambda)$ we can look at $\mathfrak{F}$ as a map $\tilde{F}: \mathfrak{S} \rightarrow \mathfrak{S}=L^{2}\left(Z, d u_{1} d u_{2} d m(\xi)\right)$. In explicit terms, 
$\tilde{F}=R F_{2} \mathcal{F}_{3}$, where $\mathcal{F}_{j}$ is the partial Fourier transform in the jth coordinate and $\mathbb{R}: \mathfrak{Q} \rightarrow \widetilde{\mathfrak{Q}}$ is given by

$$
(\Omega \phi)\left(u_{1}, u_{2}, \xi\right)=\phi\left(u_{2}-u_{1},-\xi\left(u_{1}+u_{2}\right), \xi\right) .
$$

THEOREM 4. (i) Each $c \in C$ acts as an automorphism of $Z$ by $z \tilde{c}=c^{-1} z c$, and hence acts dually on $U(\cdot, \lambda)$ by the definition $\left({ }^{\circ} U\right)(z, \lambda)=U(z z, \lambda)$ for all $z \in Z$. Moreover, for each fixed $\lambda$ and $c,{ }^{\circ} U(\cdot, \lambda)$ and $U\left(\cdot, \lambda \alpha^{2}\right)$ are unitarily equivalent: $i . e .$, there exists an operator $S(c, \lambda)$, unique up to scalars, such that ${ }^{\circ} U(z, \lambda)=S(c, \lambda)^{-1} U\left(z, \lambda \alpha^{2}\right) S(c, \lambda)$ for all $z$.

(ii) For $c=c(a, 1)$, set $S(c, \lambda)=V(a, \lambda)$. Then the arbitrary scalar for $V(a, \lambda)$ may be fixed in such a way that $a \rightarrow V(a, \lambda)$ is a unitary representation of $\mathrm{SL}(2, \mathrm{C})$. In fact, the operators $V(a, \lambda)$ are given explicitly on a set of generators for $\operatorname{SL}(2, C)$ as follows: For $f \in \mathfrak{M}$,

$$
\begin{aligned}
(V(v(k), \lambda) f)(t) & =\exp \left\{-i \operatorname{Re}\left(\lambda t^{2} k\right)\right\} f(t), \\
(V(l(b), \lambda) f)(t) & =|b| f(t b), \\
(V(p, \lambda) f)(t) & =|\lambda| f(\lambda t),
\end{aligned}
$$

where

$$
v(k)=\left(\begin{array}{ll}
1 & k \\
0 & 1
\end{array}\right), \quad l(b)=\left(\begin{array}{ll}
b & 0 \\
0 & b^{-1}
\end{array}\right), \quad p=\left(\begin{array}{rr}
0 & -1 \\
1 & 0
\end{array}\right) \in \operatorname{SL}(2, C)
$$

with $k \in C$ and $b \in C^{*}$, and

$$
\hat{f}(t)=\pi^{-1} \int_{C} f(w) \exp \{2 i \operatorname{Re}(t w)\} d w .
$$

(iii) For $c=c(I, \alpha)$ the operators $S(c, \lambda)$ are independent of $\lambda$ and we can set $S(c, \lambda)=D(\alpha)$, where for $f \in \mathfrak{M}$ we have $(D(\alpha) f)(t)=|\alpha| f(t \alpha)$. $D(\alpha)$ intertwines the representations $V(\cdot, \lambda)$ and $V\left(\cdot, \lambda \alpha^{2}\right)$ of $\operatorname{SL}(2, C)$. In particular, the representations $V(\cdot, \lambda)$ are all mutually unitarily equivalent.

(iv) The commuting algebra of $V(\cdot, \lambda)$ is two-dimensional and the operators $D(1)=I$ and $D(-1)$ form a basis.

Lemma 5. Let $\hat{T}(h, \chi)=\mathfrak{F} T(h, \chi) \mathfrak{F}^{-1}$ for all $h \in H$. Then for $\phi \in \mathfrak{W}$ and $z \in Z, a \in \operatorname{SL}(2, C), \alpha \in C^{*}$, one has

$$
\begin{aligned}
(\hat{T}(z, \chi) \hat{\phi})(\lambda) & =\hat{\phi}(\lambda) U\left(z^{-1}, \lambda\right), \\
(\hat{T}(c(a, 1), \chi) \hat{\phi})(\lambda) & =V(a, \lambda) \hat{\phi}(\lambda) V(a, \lambda)^{-1}, \\
(\hat{T}(c(I, \alpha), \chi) \hat{\phi})(\lambda) & =|\alpha|^{--4}[\alpha]^{m} D(\alpha) \hat{\phi}\left(\lambda \alpha^{-2}\right) D(\alpha)^{-1} .
\end{aligned}
$$

Lemma 6. The bounded operator $\hat{B}$ commutes with $\hat{T}(h, \chi)$ for all $h \in H$ 
if and only if there exists a measurable function $\lambda \rightarrow \hat{B}(\lambda)$ with values in bounded operators on $\mathfrak{M}$ such that the following three conditions hold:

(1) For each $\phi \in \mathfrak{W},(\hat{B} \hat{\phi})(\lambda)=B(\lambda) \hat{\phi}(\lambda)$ a.e.

(2) For each $a \in \mathrm{SL}(2, C), \hat{B}(\lambda) V(a, \lambda)=V(a, \lambda) \hat{B}(\lambda)$ a.e.

(3) For each $\alpha \in C^{*}, \hat{B}\left(\lambda \alpha^{-2}\right)=D(\alpha)^{-1} \hat{B}(\lambda) D(\alpha)$ a.e.

THeOREM 7. (i) $B$ commutes with $T(h, \chi)$ for all $h \in H$ if and only if $\hat{B}$ satisfies condition (1) above and there exist constants $c_{1}$ and $c_{2}$ such that $\hat{B}(\lambda)=c_{1} I+c_{2} D(-1)$ for a.e. $\lambda$.

(ii) Let $\hat{J}$ be the commuting operator defined for $\hat{\phi} \in \hat{\mathfrak{S}}$ by $(\hat{J} \hat{\phi})(\lambda)$ $=D(-1) \hat{\phi}(\lambda)$. Viewed as an operator on $\tilde{\mathfrak{E}}$, $\hat{J}$ becomes $\tilde{J}$, defined by $(\tilde{J} \tilde{\phi})\left(u_{1}, u_{2}, \xi\right)=\tilde{\phi}\left(-u_{1}, u_{2}, \xi\right)$ for $\phi \in \tilde{\mathfrak{S}}$.

(iii) With $J$ as in Theorem $1, J=\tilde{\mathfrak{F}}^{-1} \mathcal{J} \tilde{F}$.

Thus Theorem 1 is established.

Dartmouth College, Hanover, New Hampshire 03755 